\title{
An old game, new experience: exploring the effect of players' personal gameplay history on game experience
}

\author{
Yavuz Inal ${ }^{1}$. Jo Wake ${ }^{2}$
}

Accepted: 9 February 2022

(c) The Author(s) 2022

\begin{abstract}
This experimental study explores how game experience differs between players with different gameplay histories within the same game universe. We are interested in how prolonged engagement with a game series affects the gameplay experience in relation to the most recent game version in the series. A total of 54 participants were divided into four groups depending on their gaming experience, namely non-gamers, new-gamers, old-gamers and core-gamers. They played the mobile version of Super Mario Run, and questionnaire data was collected after the gameplay session. The results of the study showed that not only the players' personal gameplay history but also the length of experience or degree of familiarity with the game universe affected the experience of playing a new game in the same game universe. Additionally, familiarity with the game universe had a positive impact on the feeling of competence, immersion, emotions and flow.
\end{abstract}

Keywords Game experience $\cdot$ Player experience $\cdot$ Gameplay history $\cdot$ Long-term engagement $\cdot$ Game usability $\cdot$ Super Mario Run

\section{Introduction}

Game user experience is a topic of major interest in human-computer Interaction (HCI), especially in the context of user experience research, and contributes to general HCI research with topics such as how to sustain motivation, support behaviour change and opportunities to explore new interaction techniques [7]. The fields of $\mathrm{HCI}$ and game research have also influenced each other over the last decade [8]. Game user research [60] is focused on measuring, analyzing and understanding players' experience and preferences [21], and evaluation of game experience involves the adaption of traditional HCI evaluation techniques and shifting the focus from productivity to enjoyment and entertainment [45]. Game experience is described by experience qualities, such as flow, engagement, presence, immersion, and fun [6]. It is essential to understand experience with

Yavuz Inal

yavuz.inal@ntnu.no

1 Department of Design, Faculty of Architecture and Design, Norwegian University of Science and Technology, Gjøvik, Norway

2 Digital Systems, Norce Energy and Technology, Bergen, Norway games in order to create good games; and building this understanding is a necessary part of the game design process [47]. Evaluation techniques vary from the direct measurement of biometric data to capture players' emotional responses [55] to the construction of subjective data through interviews and focus groups or the use of standardized questionnaires [20].

In addition to understanding how to design engaging and enjoyable gaming experiences, our research has implications for how media-specific experiences are related to personal transformation and development over time, and additionally touches on the subject of nostalgia [27] in games. When players engage with games of the past it is often labelled retrogaming or classic gaming [71]. Like with film, literature and music, games are embedded in our culture, and are also related to our emotional and cognitive development [43], and interaction with and memories of these form part of our development as humans. Wulf et al. [72] have studied the effects of retrogaming on psychological well-being and found for example that it is positively connected with feelings of social connectedness and competence. Whilst retrogaming or classical gaming involves engaging with old games through collection of old gaming devices, or playing old games ported to new platforms, this study differs from retrogaming in that we seek to understand how previous 
experience with a game shapes the gaming experience with a new version of a game within the same game universe.

Some studies in game research have adopted a longitudinal study design [i.e., 1, 44 or explored long-term engagement in long games where a single gameplay session lasts for more than one hour [i.e., 24; however, we were unable to identify any studies concerned with the influence of longterm engagement and sustained interaction with a specific game universe on experience with new versions of the game. This study addresses this research gap by exploring how experiences differ in a game when players have different gameplay history in the same game universe. Gameplay history refers to the length of experience and degree of familiarity with a game universe. We were interested in the significance of long-term engagement within a game universe and determining how sustained experiences with the evolution of a game series affect game experience with the most recent version of an old game. We also examined the effect of game experience on perceived usability as the usability of a game contributes to the overall game experience.

\section{Literature review}

\subsection{Game usability}

Measuring the usability of a game provides critical understanding of how players approach the game, how they interact with the game interface, and what the potential hurdles toward a good quality gaming experience are [54]. Game usability, defined as "the degree to which a player is able to learn, control, and understand a game" [51], p. 1453], is more elusive to measure. It affects quality of the interface design and player engagement in games [24]. Game usability can be considered to be a complementary factor that has the potential to contribute to one of the most important marker components of game experience, flow [2], and be correlated to fun and enjoyment [63]. Visual elements are central to game usability which addresses the game interface presented between the player and the game itself [24]. The frustration of poor visual design and aesthetics of a game lowers ratings on users' perception of usability [67] which indicates that usability deficiencies may negatively affect the experience of flow and immersion [46], and positive and negative emotions [2].

\subsection{User experience evaluations in games}

The phenomenon of player experience is multidimensional and research on game experience has made remarkable progress in recent years. Studies exploring the impact of different factors on the quality of game experience and players' ingame performance show that game experience is influenced by various sources. These include visual distractors [17], leaderboard manipulations [10], reward types [50], the genre of the game [35], game platform [2, 3, 38], the personality of the player [36], the presence of social entity [40], and peak-end effects [29]. Gameplay data [41], psychophysiological signals, e.g., facial expression and electrodermal activity [64], and computational models of players' intrinsic motivation [28] have also been analyzed to either predict or understand players' experiences. Behavioral theory has been applied to interpret the results related to, for example, the role of attribution [21] and motivation [18].

The degree of the challenge in the level [65] and the challenge-skill balance [37] affect player experience in a positive way and is required to be considered for optimal game experience. Self-reported challenge is correlated with the experience of immersion and flow during gameplay [61]. When the difficulty provided is reflective of their gameplay experience, players achieve a higher immersion level [15]. Gaming frequency and past gaming experience are, therefore, also linked to the achievement of goals. Experienced players who play games for $3 \mathrm{~h}$ or more per week have more advantage in perceptual and spatial attentional skills when compared to novice players who play games for $1 \mathrm{~h}$ per week [5].

\subsection{Long-term engagement: expertise, skill, familiarity}

Past gaming experience is usually considered as a subjective relationship between the player and the game [13]. It addresses a long-term engagement in a game which can be defined as "the degree of voluntary use of a system along a wide period of time (i.e., weeks, months, or years), involving dozens, if not thousands, of interactions, each one spanning for significantly longer than a few seconds or minutes" [24], p. 4063]. Past gaming experience influences abstraction, problem-solving and user interactivity [14] and the learning of new games. Several game experience models emphasize this personal relationship between time and experience as having an effect on game experience [e.g. 23, 25.

Few studies deal with the effect of long-term engagement with a game universe on aspects of game usability specifically. Recently, the theme of how to design for sustained engagement, particularly in the area of health behaviour change, has become popular. There are also several game usability studies that consider player expertise, which is one aspect of long-time engagement. Iacovides et al. [31], for example, study the role of player expertise in relation to game experience, and how the skill level of the player affects the level of enjoyment, and striking the balance between game challenge and player competence. Building on Galloway's [26] distinction between diegetic (direct interaction with the game, viewed by the game character) 
and non-diegetic (player support, viewed by the player) elements, they argue that skilled players prefer less non-diegetic elements in order to experience game immersion. Although there is substantial evidence that the players' expertise impacts experience with a particular game, and that the skill-challenge balance is an important aspect when designing for immersive experiences, we argue that skill, mastery and expertise is one aspect of long-term engagement, but does not cover everything. Long-term engagement with a game series is more multi-faceted, involving familiarity of the game characters, aesthetics, memories of playing and social connections to peer players for example.

The designer of Pokémon, Satoshi Tajiri has explained how the idea for the game originated with his childhood fascination for catching insects [66], and imagining insects travelling through cables connecting the Game Boy devices as a form of communication and exchange with peer players. Building on Shields and Johnson's [62] construct of childhood brand nostalgia (CBN), defined as " $[\ldots]$ a positively valenced emotional attachment to a brand because of the brand's association with fond memories of the individual's non-recent lived past" [p. 346], Harborth and Pape [30] explored how nostalgia affects technology acceptance with Pokémon Go, a subsequent game in the same universe as the original Pokémon. Using the Unified Theory of Acceptance and Use of Technology adapted for consumers (UTAUT2) and voluntary technology use [69], they found positive correlations between $\mathrm{CBN}$ and both behavioural intentions to adopt and play Pokémon Go and actual use, and particularly the model elements of effort expectancy, hedonic motivation and facilitating conditions. While these findings are relevant to our study, our focus is on usability rather than technology acceptance.

\subsection{This study}

Taken together, these studies support the notion that there is a plethora of game experience evaluations and several approaches to conducting them. However, we had difficulty finding other relevant works to cite considering the lack of studies specifically studying game experience related to previous, long-time exposure to a game franchise and effect of usability on such experience. Furthermore, the existing research is insufficient to explain how game usability correlates with game experience related to previous exposure to a game franchise. This paper, therefore, contributes to HCI research on game experience evaluation by studying how long-term engagement within a game universe affects user experiences with new games within the same universe. The following research questions guided the study:

- How does the presence or absence of players' personal gameplay history impact on their game experience?

- How do the length of experience and degree of familiarity with a game universe influence game experience and perceived usability?

- What is the relationship between game experience and perceived usability for players with different gameplay histories?

- What is the role of perceived usability in predicting game experience?

\section{Methods}

An experimental study was conducted based on a gameplay session after which data was collected through questionnaires. The following sections provide information about the study participants, information about the questionnaires, Super Mario Run game and data collection process.

\subsection{Participants}

The participants were recruited from a pool of about two hundred students taking an undergraduate course on HCI. The recruitment method was purposive sampling, using the email list for the course. The following inclusion criteria were used: (1) being a casual game player that enjoys any mobile games without investing significant time to it, playing mobile games irregularly or infrequently, (2) having a mobile phone and at least two years of experience with touch-based interaction, (3) had never played the original Super Mario Run game developed by Nintendo Co. Ltd. on their mobile phones at the time when the study was conducted, and (4) fitting one of the categories given in Table 1. These criteria were used to control the variable of gameplay

Table 1 The participants' gameplay history in the Super Mario game universe

\begin{tabular}{lll}
\hline Player type & Gameplay history & Frequency \\
\hline Non-gamers & I have never played a Super Mario game franchise before & 12 \\
New gamers & I have played a Super Mario game franchise on a PC/game console within the last two years & 15 \\
Old gamers & I have played a Super Mario game franchise on a PC/game console several times but not within the last two & 13 \\
& years & I have been playing a Super Mario game franchise on a PC/game console for more than five years \\
Core gamers &
\end{tabular}


history in the Super Mario game universe. A total of 54 university students, 33 male and 21 female, participated in the study. The mean age of the participants was 23.8 years $(\mathrm{SD}=2.78)$.

In accordance with the purpose of the study, the participants with different personal gameplay histories in the Super Mario game universe were selected. We recruited the participants from those that either had no experience in playing any Super Mario game or had played at least one game from this series. The participants were compared according to their gameplay history of Super Mario games: those with no previous experience with Super Mario games (nongamers); those who played a Super Mario game recently (new gamers), those who previously played a Super Mario game but stopped (old gamers); and those who are playing a Super Mario game for more than five years (core gamers), as shown in Table 1. All participants self-identified themselves as a "casual" gamer, which means that gameplay time or interest in gaming is limited.

Since the whole Super Mario game series has similar goals related to controlling the same game character(s), we did not specify the game version. However, core gamers and old gamers were familiar with non-platform games of the Super Mario franchise (e.g., Mario Kart, Super Smash Bros.) but not new gamers. All groups were familiar with endless runner games on mobile phones. Sample selection was performed via a short demographic survey containing questions about the participants' experiences with the Super Mario game universe and daily gameplay habits on mobile phones. The gameplay history of the participants was considered as the independent variable of the study and the participants' in-game experiences and perceived usability scores as the dependent variables. After gathering the results of game experience, analyses were performed on the data to explicate the relationships between the study variables.

\subsection{Data materials}

The data were collected using the Game Experience Questionnaire (GEQ) and System Usability Scale (SUS). GEQ aims to understand the digital game experiences of players [32]. This questionnaire has become one of the most commonly used measurement scales to determine the key attributes of gameplay experience $[34,48]$ and has been used in a variety of research settings in the literature to explore game experience [e.g., 2, 38, 49. The scale is a 33-item scale which aims to measure game experience across seven dimensions, namely competence, sensory and imaginative immersion, flow, tension/annoyance, challenge, negative affect, and positive affect.
Recent literature shows that some items in GEQ are vague [20] and the scale has some reliability problems, mainly for the challenge and negative affect components [39]. We, therefore, used a modified version of the scale as suggested by Johnson et al. [34] to increase reliability and validity of the findings. The modified version was presented in the following five constructs: positive affect, negativity, competence, flow, and immersion [34]. We calculated the reliability of the modified GEQ and the Cronbach alpha score was found to be 0.826 well above threshold level [9].

SUS was developed by Brooke [12] to evaluate the perceived usability of a digital product. It consists of ten items rated on a five-point Likert scale ranging from 1 ("strongly disagree") to 5 ("strongly agree") and has been used in various research settings to investigate the effect of usability on player experience [e.g., 2, 46, 58. In order to better suit the scale to our research, we tailored the scale by replacing the terms "system" and "use" with "mobile game" and "play", respectively.

\subsection{Super Mario Run}

The participants played the mobile version of Super Mario Run. Super Mario has a very long history and is one of the most well-known games in the world [19]. The game was first released by Nintendo Entertainment System in 1985 as Super Mario, and then several new versions with various themes were released by the company selling millions of copies in total [22]. The game Super Mario is one of the oldest currently active computer games in the world, and has been released for a range of different platforms and versions, and different game mechanics. Being more than 30 years old, it opens an opportunity to investigate how the long-term relationship with a game universe affects player experience. Several of the computer games being played today belong to a game series, such as Warcraft, FIFA, The Legend of Zelda or Grand Theft Auto, and game developers frequently publish games as series and franchises.

Super Mario Run was made available for iOS platforms in 2016 and for Android in 2017. It was also the first official Super Mario game that was developed for mobile devices. In the game, the main character (Mario) runs constantly and the player controls the jumping action by tapping on their mobile device in order to gather coins and reach a goal (Fig. 1). The game can be downloaded for free; however, players need to purchase it (in-app purchases) to play the full version of the game offering four modes: World Tour, Toad Rally, Remix 10, and Kingdom Builder. In this study, the full version of the game was used. 

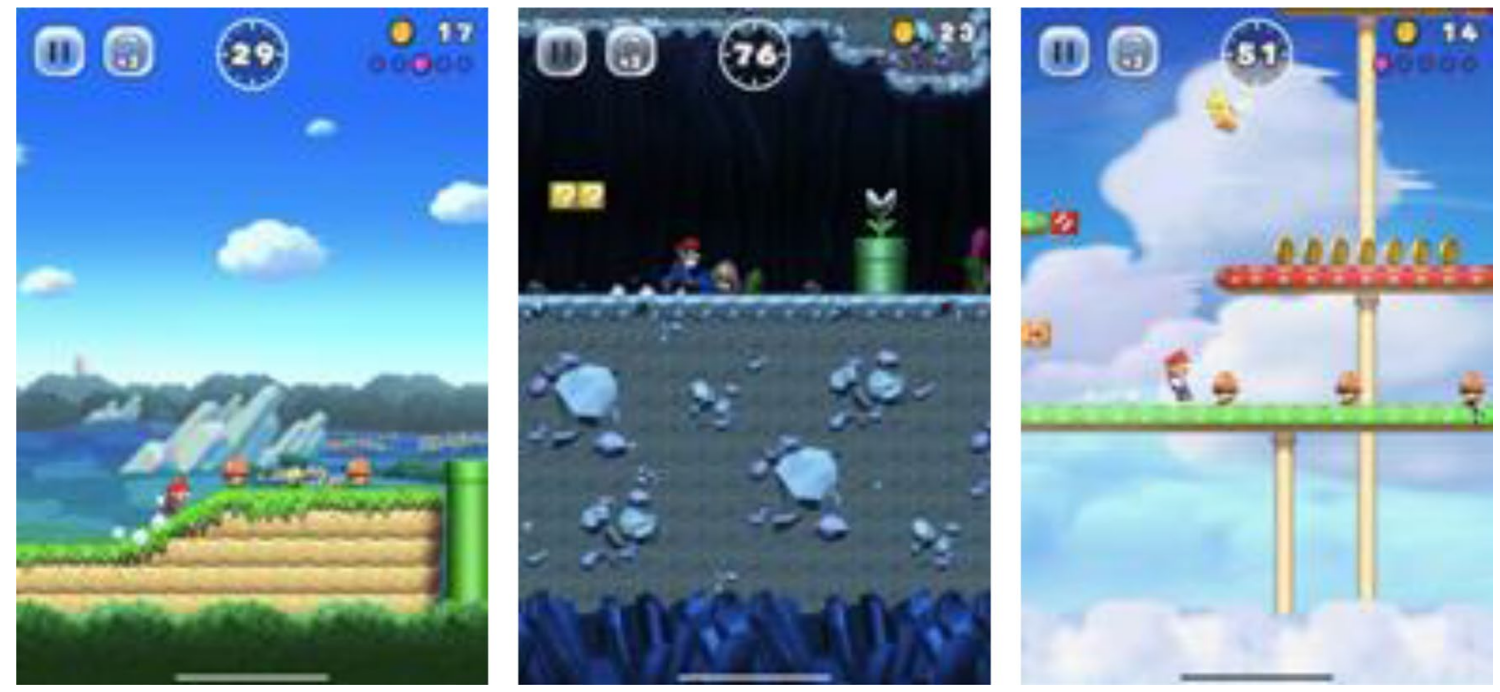

Fig. 1 Screenshots from Super Mario Run

Table 2 Game experience scores of the participants for each study variable by player type

\begin{tabular}{lllll} 
& Non-Gamers & New Gamers & Old Gamers & Core Gamers \\
\hline The Core Questionnaire & Mean (SD) & Mean (SD) & Mean (SD) & Mean (SD) \\
\hline Positive Affect & $1.67(0.88)$ & $2.68(1.00)$ & $3.26(0.92)$ & $3.51(0.90)$ \\
Negativity & $2.90(1.20)$ & $2.28(0.87)$ & $2.14(0.61)$ & $2.09(0.80)$ \\
Competence & $2.21(1.04)$ & $2.83(0.87)$ & $3.13(0.56)$ & $3.34(0.81)$ \\
Flow & $2.30(1.21)$ & $2.23(0.62)$ & $2.49(0.72)$ & $2.81(0.93)$ \\
\hline Immersion & $1.46(0.53)$ & $2.65(0.77)$ & $2.27(0.70)$ & $2.73(0.64)$ \\
\hline
\end{tabular}

Green indicates positive game experience and red represents negative game experience (inversely)

\subsection{Procedure}

The experiment was conducted in a classroom. The participants were physically isolated from others during the gameplay session that lasted $20 \mathrm{~min}$. Before starting the experiment, the moderator informed the participants about how to play the game showing a video. The participants were also informed that participation was voluntary, and they could stop and leave the experiment at any time. Considering that differences in mobile device capabilities could affect participants' game experience [2, 53], they were asked to complete the experiment using the same mobile device provided (4.7-in. iPhone 6 running iOS). The device met the device requirements to run the game properly. Two graduate students helped the participants as moderators of the experiment by informing them about the experimental procedure, explaining the main purpose of the study, and describing how to play the game before each gameplay session. Since the scale should not be completed after long gameplay sessions in which the participants may not remember their gaming experience properly [56] and needs to be administered immediately after the gameplay session [32], the participants were asked to complete the paper version of the questionnaires (modified version of GEQ and SUS) in the same sequence after each gameplay session without taking a break. 


\section{Results}

We present the results beginning with a description of game experience and perceived usability. This is followed by our findings related to interrelations between game experience and perceived usability, effect of gameplay history on game experience and perceived usability, and the role of perceived usability in predicting gameplay experience.

\subsection{Description of game experience and perceived usability}

As shown in Table 2, the participants with a long history in playing the game (core gamers) had the highest scores in all positive constructs, namely positive affect, competence, flow and immersion. Similarly, the core gamers had the lowest score in negativity. The old gamers had higher scores in positive affect, competence, and flow, and lower score in negativity than the new gamers. The new gamers had higher scores in immersion than old gamers, but their scores were lower than those of the core gamers. They had higher negativity scores than old gamers and core gamers.

The participants who had never played a Super Mario game before had the highest game experience score in negativity, whereas they had the lowest scores in most positive constructs, i.e., positive affect, competence and immersion, and the second lowest score in flow construct. The participants (from the non-gamers to core gamers) tended to be more in favor of the game when they had more experience in playing it. All constructs were ranked in an order in line with their experience, with the exception of flow, for which the new gamers scored the lowest, and immersion for which the old gamers scored the second lowest.

Based on the descriptive outcomes of gameplay experience, the results indicated that not only the presence of players' personal gameplay history but also the length of experience or degree of familiarity with the game universe influenced game experience in the same game universe. The perceived usability score of the game was the highest among the old gamers $(M=66.35, S D=11.66)$, followed by the core gamers $(M=65.54, S D=13.20)$, new gamers $(M=55.33, S D=17.29)$, and non-gamers $(M=48.33$,
$\mathrm{SD}=13.71)$. While the old and core gamers had nearly the same evaluation score, the game was considered to be least usable by the non-gamers.

\subsection{Interrelations between game experience and perceived usability}

Table 3 demonstrates the correlation between game experience and perceived usability for each player type. The results showed that the perceived usability score of the non-gamers was strongly correlated with the highest number of game experience constructs, namely negativity, competence, and flow. This group was followed by the new gamers, old gamers, and finally core gamers. Among the correlated constructs, only competence was significantly and positively correlated with the perceived usability score. The remainder of the constructs were significantly and negatively linked to the perceived usability score. This indicates that among the non-gamers, perceived usability increased when the competence score increased, but all the other correlated item scores decreased.

The perceived usability score of the new gamers was significantly associated with the three game experience constructs: positive affect, negativity, and competence. However, the perceived usability score of the old gamers was significantly correlated with only two game experience constructs: negativity and competence. This indicated that usability perception was correlated with more constructs in the non-gamer and new gamer groups compared to the old gamers and core gamers. Besides, negative opinions toward the usability of the game reinforced the negative aspects of experience, such as negativity among the non-gamers at a higher level compared to the new gamers, old gamers and core gamers.

Positive affect and flow were the significantly least correlated constructs with perceived usability among all player types. Flow was significantly and negatively correlated with only the perceived usability scores of the nongamers, whereas positive affect was linked to the usability perception of the new gamers. However, negativity and competence were significantly most correlated constructs with perceived usability among the participants. Negativity
Table 3 Interrelation between game experience and perceived usability

\begin{tabular}{lllll}
\hline The Core Questionnaire & Non-gamers & New gamers & Old gamers & Core gamers \\
\hline Positive affect & 0.20 & $0.61^{*}$ & 0.49 & 0.50 \\
Negativity & $-0.84^{* *}$ & $-0.60^{*}$ & $-0.57^{*}$ & $-0.64^{*}$ \\
Competence & $0.79^{* *}$ & $0.56^{*}$ & $0.56^{*}$ & 0.35 \\
Flow & $-0.73^{* *}$ & 0.17 & 0.32 & 0.07 \\
Immersion & -0.09 & 0.34 & -0.02 & 0.40 \\
\hline
\end{tabular}

$* p<0.05, * * p<0.001$ 
Table 4 Test results demonstrating the influence of player types on the study variables

\begin{tabular}{llll}
\hline Study variables & $F$ & $P$ & Post-hoc comparisons $^{\text {a }}$ \\
\hline Positive affect & 9.921 & 0.000 & NoG $<$ NG, NoG $<$ OG, NoG $<$ CG, NG $<$ CG \\
Competence & 4.413 & 0.008 & NoG $<$ OG, NoG $<$ CG \\
Immersion & 9.565 & 0.000 & NoG $<$ NG, NoG $<$ OG, NoG $<$ CG \\
Perceived usability & 4.707 & 0.006 & NoG $<$ OG, NoG $<$ CG, NG $<$ OG \\
\hline
\end{tabular}

$N o G$ non-gamers, $O G$ old gamers, $N G$ new gamers, $C G$ core gamers

${ }^{\mathrm{a}}<$ reflects a significant difference

$p<0.001$
Table 5 Linear regression analyses between perceived usability and change in game experience scores

\begin{tabular}{|c|c|c|c|c|c|c|c|c|}
\hline \multirow[t]{2}{*}{ Model } & \multicolumn{2}{|c|}{ Non-gamers } & \multicolumn{2}{|c|}{ New gamers } & \multicolumn{2}{|c|}{ Old gamers } & \multicolumn{2}{|c|}{ Core gamers } \\
\hline & $\beta$ & $R^{2}$ & $\beta$ & $R^{2}$ & $\beta$ & $R^{2}$ & $\beta$ & $R^{2}$ \\
\hline Positive affect & - & - & 0.035 & 0.372 & - & - & - & - \\
\hline Negativity & -0.074 & 0.706 & -0.030 & 0.362 & -0.030 & 0.322 & -0.039 & 0.415 \\
\hline Competence & 0.059 & 0.617 & 0.028 & 0.309 & 0.027 & 0.308 & - & - \\
\hline Flow & -0.064 & 0.531 & - & - & - & - & - & - \\
\hline Immersion & - & - & - & - & - & - & - & - \\
\hline
\end{tabular}

was significantly and negatively associated with all player types, whereas competence was significantly and positively linked to the non-gamers, new gamers and old gamers. Only immersion was not correlated with any of the player types.

\subsection{Effect of gameplay history on game experience and perceived usability}

After meeting the related assumptions, a series of one-way analysis of variance (ANOVA) tests were performed on the data for each of the four player types in order to determine whether the participants' gameplay history affected their game experience. The significant results of ANOVA and post-hoc tests are presented in Table 4. The participants' gameplay histories had a statistically significant effect on their game experience, specifically positive affect $\left[F(3,50)=9.921, p=0.000, \eta^{2}=0.371\right]$, competence $[F(3$, $\left.50)=4.413, p=0.008, \eta^{2}=0.209\right]$, and immersion $[F(3$, $\left.50)=9.565, p=0.000, \eta^{2}=0.365\right]$. However, there were no significant differences in negativity $[F(3,50)=2.237$, $p=0.095]$ and flow $[F(3,50)=1.237, p=0.306]$ constructs. Besides, the player type statistically significantly affected the participants' scores in perceived usability $[F(3,50)=4.707$, $\left.p=0.006, \eta^{2}=0.220\right]$.

The post-hoc tests revealed that the participants' gameplay history was found to significantly affect their positive game experience. For instance, the non-gamers had significantly lower positive affect scores compared to the old gamers $(p=0.000)$, new gamers $(p=0.007)$, and core gamers $(p=0.000)$. Core gamers had significantly higher positive affect scores than new gamers $(p=0.019)$. Concerning competence, the non-gamers had significantly lower scores than the old gamers $(p=0.008)$ and core gamers $(p=0.001)$. Regardless of the length of experience in playing the game, the participants had significantly higher immersion scores than those that had never played a Super Mario game before (core gamers, $p=0.000$; old gamers, $p=0.004$; new gamers, $p=0.000)$.

In addition to the participants' game experience, their perceived usability scores were significantly influenced by the length of experience or degree of familiarity with the game universe. The post-hoc tests revealed that the old gamers $(\mathrm{M}=66.35, \mathrm{SD}=11.66, p=0.003)$ and core gamers $(M=65.54, S D=13.20, p=0.003)$ considered the game to be more usable than the non-gamers $(M=48.33$, $\mathrm{SD}=13.71)$; however, the new gamers $(\mathrm{M}=55.33$, $\mathrm{SD}=17.29)$ considered the game to be less usable than the old gamers $(p=0.047)$.

\subsection{The role of perceived usability in predicting gameplay experience}

To explore the possible predictive value of the perceived usability on the player experience, we performed linear regressions with the perceived usability as predictor variables and with the game experience constructs as dependent variables. Table 5 demonstrates analysis results between perceived usability and change in game experience scores for each player type. Regression modeling showed that perceived usability explained significantly variation in all game experience constructs, with the exception of immersion.

Perceived usability significantly predicted negativity for all player types. The model explained $70.6 \%(R 2=0.706)$ of the variance for non-gamers $[F(1,10)=24.042$, 
$p=0.001] ; 36.2 \%(R 2=0.362)$ of the variance for new gamers $[F(1,13)=7.387, p=0.018] ; 32.2 \%(R 2=0.322)$ of the variance for old gamers $[F(1,11)=5.220, p=0.043]$; and $41.5 \%(R 2=0.415)$ of the variance for core gamers $[F(1,12)=8.522, p=0.013]$.

There was a collective significant effect between perceived usability and competence for all player types, with the exception of core gamers. The individual predictors were examined further and indicated that perceived usability predicted competence score for non-gamers $[F(1,10)=16.090$, $p=0.002)$; new gamers $[F(1,13)=5.816, p=0.031]$; and old gamers $[F(1,11)=4.904, p=0.049]$. The model significantly explained $61.7 \%(R 2=0.617), 30.9 \%(R 2=0.309)$, and $30.8 \%(\mathrm{R} 2=0.308)$ of the variance, respectively. Results of linear regression also showed that there was a significant effect between perceived usability and positive affect for only new gamers $[F(1,13)=7.694, p=0.016, \mathrm{R} 2=0.372]$ and flow for only non-gamers $[F(1,10)=11.320, p=0.007$, $\mathrm{R} 2=0.531]$.

These results indicated that perceived usability predicted the game experience, and explained variance was very high for negativity, competence, and flow for non-gamers, respectively. The model significantly explained the variance of the positive affect for only new gamers and flow for only nongamers. Only the negativity construct was predicted by perceived usability for core gamers, negativity and competence constructs were predicted for old gamers. This indicates that higher perceived usability might lead to better game experience results for specifically non-gamers and new gamers when compared to old gamers and core gamers.

\section{Discussion}

The field of computer games is steadily advancing, with more and more games becoming available every day; however, numerous issues that may affect gameplay experience remain unexplored. Since players are at the center of this experience, player types should be seen as a potential factor that can influence game experience. Previous studies [e.g., 2, 52 suggested that researchers should examine the relationship between player types, player motivation, and game experience. Thus, this experimental study aimed to explore how gameplay experiences differed between players with different gameplay histories in the same game universe. We also examined how game experience influenced the participants' perceived usability scores. The main finding in our study was that the length of experience or familiarity with a game universe affected the individuals' experience of playing a game. Additionally, familiarity with the game universe had a positive impact on the feeling of competence, immersion, emotions, and flow.
The descriptive measures of central tendency showed that the absence of personal gameplay history affected the players' game experience in the same game universe. For instance, the non-gamers with no experience in playing a Super Mario game had evidently different gameplay experience when compared to those that had played this game before. Not only did they have the lowest score in experiences related to competence, immersion, and positive affect, but they also had the highest score in negativity. More specifically, the non-gamers were not able to immerse in the game. The immersion construct refers to being able to identify with avatars, aesthetics, and narrative parts of a game, using imagination to enter a fantasy world, and having rich experiences other than merely seeing gameplay as a challenge, and the non-gamers were far from experiencing these aspects of the game. This distance may also be related to the experience of flow, which was also low for the non-gamer group. At the same time, the presence and length of previous experience makes it easier for players to immerse in the game, which is why the core gamers in this study scored high in game immersion. Similarly, Engl and Nacke [23] found that hardcore gamers were less prone to distractions and were more easily immersed in the game.

Interestingly, the non-gamers expressed feelings of annoyance and frustration with the game in line with their experience of distance. The new gamers were more annoyed than the old gamers, reporting feeling a low level of competence, a certain experience of flow, and low positive affect. One reason for this could be that the new gamers did not enjoy playing a Super Mario game on a mobile device since they were familiar with the computer version. These findings can also be considered to indicate that players experience various emotions simultaneously while playing a game. Examining gaming experience on mobile devices, Engl and Nacke [23] found that mobile gameplay was influenced by several contextual factors, including spatial, temporal, social, cultural, and psychological. Playing games whilst embedded in a context that is not exclusively organized for gaming has an effect on gameplay experience. Furthermore, considering that the old gamers had more experience with the Super Mario game universe, but they had stopped playing the game for a while, these findings might also be related to these players having previously completed the game.

The experiences related to positive and negative emotions revealed the largest polarities between the non-gamers and core gamers, as shown in Table 2. We found statistically significant differences between these groups in terms of negative emotions (Table 3). The presence or absence of a gameplay history within the game universe seems to have affected the emotional outcome of gameplay. After the Super Mario Run experience, the core gamers reported strong positive emotions, such as having fun, being content, and 
feeling happy, which indicates that a history of familiarity with a game universe leads to a positive emotional outcome when playing a new version of the game. Where there is an absence of such history, there is an increased range of possible outcomes regarding emotions.

The absence of game universe experience was also found to affect immersion. In this respect, the non-gamers were significantly different from the remaining three groups. One explanation for this is that to reach the level of immersion in a game, a player must be able to easily operate in the game, in line with the [31] discussion of skilled gamers being able to play without the presence of non-diegetic interface elements. Despite involving a very simple interaction through tapping the screen, Super Mario Run requires motor skills and timing built through repetitive practice and developing a rhythm for the game, which the non-gamers did not necessarily have. There was also a significant difference in reported game competence between the non-gamers and the old and core gamers. This indicates that the players were able to transfer their gameplay skills between platforms, such as consoles, personal computer, and mobiles, and different versions of the Super Mario game. The results also showed that the length of experience or degree of familiarity with the game universe influenced the current game experience. The core gamers, who had played Super Mario continuously for at least five years before the study, had different immersion than the old gamers that had stopped playing Super Mario at least two years before. The positive emotions of the core gamers also significantly differed from those of the new gamers and non-gamers.

The old gamers and core gamers found the game to be more usable than the other groups, although their scores were around the cut-off points. In the literature, studies suggest that an average usability score of below 70 indicates poor usability of the evaluated system and represents low quality of usability [e.g., 4, 42, 68. For the non-gamers and new gamers, the scores were far below the cut-off points, indicating that Super Mario Run for mobile devices does not have a particularly usable interface. In line with the above-mentioned findings related to competence, the players with previous experience were able to transfer it to the new platform, overcoming problems with using the interface. However, considering the cut-off values, regardless of the participants' previous game experience, the game was not evaluated to be highly usable.

There was a significant and negative correlation between perceived usability and negativity (including negative affect, challenge and tension/annoyance) for all player types (Table 4); i.e., the more challenging the game was, the less usable it was for the players. Interestingly, the core gamers found the game challenging, but usable at the same time. The discrepancy of how challenge affects usability perceptions can be captured by Salen and Zimmerman's [57] description of one of the most central game design challenges: striking the balance between the challenge for and the ability of the intended user. When a challenge is presented as a problem to be dealt with, a user experiences anxiety until that problem is overcome. When a problem or task is perceived as too easy, the user can experience boredom. Problems that are perceived as impossible to solve lead to boredom and quitting. Furthermore, challenge and skills present a dynamic pair, in which tackling the former increases the player's ability and skills [70]. Challenges should thus have an increasing level of difficulty and novelty throughout the game, with carefully designed levels to include novice gamers [11]. Salen and Zimmerman's concept of the interplay of challenge and skills identifies one of the inherent challenges of game design [57].

There is a strong and positive correlation between the competence and usability perceptions for all player types, with the exception of core-gamers. Although core gamers had the highest competence score, usability of the game did not correlate with this construct which refers to how players feel competent/successful/skillful/good at the game. There is also a significant and negative correlation between the flow and usability perceptions of the non-gamers. Flow is related to the competence level of players playing a game; thus, it refers to any challenge or obstacle that a player encounters during gameplay [16] and affects the overall experience [33]. One obstacle to feeling the flow in a game is finding the game boring, which was also significantly identified for the non-gamers in this study. Boredom can also affect usability perceptions that are closely associated with remaining engaged in the game to achieve mastery. In this study, the non-gamer group's high scores in boredom were considered to have led to such lack of engagement in order to achieve mastery.

Game usability comprises good aesthetics and interactive design features of interface and navigation. There are still several games which are released with plenty of serious usability problems despite the fact that usability of a game and the quality of its user interface are very important for players [59]. Game usability affects not only quality of player engagement with a game [24] but also player experience [2, 63]. Similarly, perceived usability, in the current study, significantly predicted most of the constructs. Negativity was predicted by perceived usability for all gamer groups whilst positive affect was predicted for only new gamers. A higher perceived usability might lead to better game experience results for specifically non-gamers and new gamers when compared to old gamers and core gamers. The main motivation of game companies, today, is to draw the attention of as many players as possible. This makes game usability a necessity rather than an ordinary effort when designing and developing a game due to peculiar features of the context. 


\subsection{Practical implications}

In this study, we experimentally explored the effect of gameplay history in a game on player experience. The ability to accurately understand player experience has implications for developing more enjoyable games. One of the main outcomes of the study was that not only the presence of players' personal gameplay history but also the length of experience or degree of familiarity with a game universe affected the experience of playing a new game in the same game universe. The participants that had never played the game had the least positive and most negative experiences while playing the game, and they also had the lowest perceived usability. This indicates that game developers should be aware of the negative attitudes of newcomers and put more effort to improve the positive game experience of this player group in order to encourage them to keep playing their games in the future. To support motivation and encourage players for long-term engagement with a game, game mechanisms should be built on the concepts of various game experience constructs such as competence, flow, immersion, challenge, and feedback.

Our results also suggest that game researchers should be careful with gathering data from people with different lengths of experience or degrees of familiarity with a game universe. Researchers should keep in mind that the results of their studies may be affected by the participants' gameplay histories varying from no previous experience to different levels of familiarity. Therefore, participants with similar demographics should be selected to prevent the potential effect of different variables on research outcomes; i.e., researchers should control as many factors as possible in order to achieve reliable and valid results.

\section{Conclusion}

Difference in experience according to previous exposure to a game franchise could intuitively be expected, however, it is important to have studies showing empirical evidence of it. The main contribution of this experimental study is, therefore, to start to uncover how the length of experience and degree of familiarity with a game universe affects the experience of playing a new game within the same game universe. The players that were familiar with the game universe, particularly those that were recently playing the games within that universe, scored high in several gaming measures. For the participants without any experience and those with previous experience that no longer played any game within that universe, the findings were more diverse and less predictable. From the perspective of the gaming industry, these findings can be interpreted in line with the notion of lack of predictability in what makes a game popular and widespread, the risk of putting enormous resources into developing the first version of a game, and explain why game development companies make games into a series if the first version becomes popular, similar to the current practices in the mainstream film industry.

The generalizability of these results is subject to certain limitations. For instance, the main weakness of this study was the paucity of the sample size. It is important to have a sufficient number of participants in order to conclude a valid research result. The contribution was based on user data captured from questionnaires. Using only quantitative data helps to find numbers and tendencies but not the real reasons for that phenomenon. To be able to understand the underlying reasons of users' behaviors, it is important to gather qualitative data. The participants were casual players who had some previous experience with the Super Mario universe. This research has, therefore, thrown up many questions in need of further investigation. Further work is needed to fully understand the implications of other types of players/game genres/platforms on game experience.

Author contributions The authors contributed equally.

Funding Open access funding provided by NTNU Norwegian University of Science and Technology (incl St. Olavs Hospital - Trondheim University Hospital). Not applicable.

Availability of data and material Materials are available.

Code availability Not applicable.

\section{Declarations}

Conflict of interest There is no conflict of interest.

Open Access This article is licensed under a Creative Commons Attribution 4.0 International License, which permits use, sharing, adaptation, distribution and reproduction in any medium or format, as long as you give appropriate credit to the original author(s) and the source, provide a link to the Creative Commons licence, and indicate if changes were made. The images or other third party material in this article are included in the article's Creative Commons licence, unless indicated otherwise in a credit line to the material. If material is not included in the article's Creative Commons licence and your intended use is not permitted by statutory regulation or exceeds the permitted use, you will need to obtain permission directly from the copyright holder. To view a copy of this licence, visit http://creativecommons.org/licenses/by/4.0/.

\section{References}

1. Adachi, P.J., Willoughby, T.: The longitudinal association between competitive video game play and aggression among adolescents and young adults. Child Dev. 87(6), 1877-1892 (2016). https:// doi.org/10.1111/cdev.12556

2. Aker, C., Inal, Y., Rizvanoglu, K.: A multi-modal approach for evaluating player experience on different gaming platforms. Int. J. 
Mob. Commun. 18(2), 175-195 (2020). https://doi.org/10.1504/ IJMC.2020.105845

3. Aker, C., Rizvanoglu, K., Inal, Y., Yilmaz, A.S.: Analyzing playability in multi-platform games: a case study of the Fruit Ninja game. In: Marcus, A. (eds.) Design, User Experience, and Usability: Novel User Experiences. DUXU 2016. Lecture Notes in Computer Science, vol. 9747. Springer, Cham (2016). https://doi. org/10.1007/978-3-319-40355-7_22

4. Bangor, A., Kortum, P., Miller, J.: Determining what individual SUS scores mean: adding an adjective rating scale. J. Usability Stud. 4(3), 114-123 (2009)

5. Bediou, B., Adams, D.M., Mayer, R.E., Tipton, E., Green, C.S., Bavelier, D.: Meta-analysis of action video game impact on perceptual, attentional, and cognitive skills. Psychol. Bull. 144(1), 77-110 (2018). https://doi.org/10.1037/bul0000130

6. Bernhaupt, R., Eckschlager, M., Tscheligi, M.: Methods for evaluating games: how to measure usability and user experience in games? In: Proceedings of the International Conference on Advances in Computer Entertainment Technology, pp. 309-310 (2007). https://doi.org/10.1145/1255047.1255142

7. Bernhaupt, R., Isbister, K., de Freitas: Introduction to this special issue on HCI and games. Hum. Comput. Interact. 30(3-4), 195-201 (2015). https://doi.org/10.1080/07370024.2015.1016573

8. Bernhaupt, R., Mueller, F.F.: Game user experience evaluation. In: Proceedings of the $2016 \mathrm{CHI}$ Conference Extended Abstracts on Human Factors in Computing Systems, pp. 940-943 (2016). https://doi.org/10.1145/2851581.2856683

9. Bland, J., Altman, D.: Statistics notes: Cronbach's alpha. BMJ 314, 572 (1997). https://doi.org/10.1136/bmj.314.7080.572

10. Bowey, J.T., Birk, M.V., Mandryk, R.L.: Manipulating leaderboards to induce player experience. In: Proceedings of the Annual Symposium on Computer-Human Interaction in Play, pp. 115120 (2015). https://doi.org/10.1145/2793107.2793138

11. Brandse, M. \& Tomimatsu, K.: Using color guidance to improve on usability in interactive environments. In: Proceedings of HCI International. Posters' Extended Abstracts, pp. 3-8 (2014)

12. Brooke, J.: SUS-A quick and dirty usability scale. Usability Evaluation in Industry 189(194), 4-7 (1996)

13. Calvillo-Gámez, E.H., Cairns, P., Cox, A.L.: Assessing the core elements of the gaming experience. In: Bernhaupt, R. (ed.) Evaluating User Experience in Games. Human-Computer Interaction Series. Springer, London (2010). https://doi.org/10.1007/ 978-1-84882-963-3_4

14. Chng, S.I., Low, Y.C., Lee, Y.L., Chia, W.C., Yeong, L.S.: Video games: A potential vehicle for teaching computational thinking. In: Kong, S.C., Abelson, H. (eds.) Computational Thinking Education. Springer, Singapore (2019). https://doi.org/10.1007/978981-13-6528-7_14

15. Cox, A., Cairns, P., Shah, P., Carroll, M.: Not doing but thinking: The role of challenge in the gaming experience. In: Proceedings of the SIGCHI Conference on Human Factors in Computing Systems, pp. 79-88 (2012). https://doi.org/10.1145/2207676.22076 89

16. Csikszentmihalyi, M.: Flow: The Psychology of Optimal Experience. Harper \& Row, New York (1990)

17. Cutting, J.: Measuring game experience using visual distractors. In: Proceedings of the Annual Symposium on Computer-Human Interaction in Play, pp. 695-698 (2017). https://doi.org/10.1145/ 3130859.3133221

18. De Grove, F., Cauberghe, V., Van Looy, J.: Development and validation of an instrument for measuring individual motives for playing digital games. Media Psychol. 19(1), 101-125 (2014). https://doi.org/10.1080/15213269.2014.902318

19. Demaine, E.D., Viglietta, G., Williams, A.: Super Mario Bros. Is harder/easier than we thought. In: Demaine, E.D., Grandoni, F. (eds.) 8th International Conference on Fun with Algorithms (FUN 2016), Article No. 13, pp. 1-15 (2016)

20. Denisova, A., Nordin, A.I., Cairns, P.: The convergence of player experience questionnaires. In: Proceedings of the Annual Symposium on Computer-Human Interaction in Play, pp. 33-37 (2016). https://doi.org/10.1145/2967934.2968095

21. Depping, A., Mandryk, R.: Why is this happening to me? How player attribution can broaden our understanding of player experience. In: Proceedings of the 2017 CHI Conference on Human Factors in Computing Systems, pp. 1040-1052 (2017). https://doi. org/10.1145/3025453.3025648

22. Dillon, R.: The Golden Age of Video Games: The Birth of a Multibillion-Dollar Industry. CRC Press, Boca Raton (2011)

23. Engl, S., Nacke, L.E.: Contextual influences on mobile player experience-a game user experience model. Entertain. Comput. 4(1), 83-91 (2013). https://doi.org/10.1016/j.entcom.2012.06.001

24. Febretti, A., Garzotto, F.: Usability, playability, and long-term engagement in computer games. In: Proceedings of the $2009 \mathrm{CHI}$ Conference Extended Abstracts on Human Factors in Computing Systems, pp. 4063-4068 (2009). https://doi.org/10.1145/15203 40.1520618

25. Fernandez, A.: Fun experience with digital games: a model proposition. In: Extending Experiences: Structure, Analysis and Design of Computer Game Player Experience, Lapland University Press, Rovaniemi, Finland, pp. 181-190 (2008)

26. Galloway, A.R.: Gaming: Essays on Algorithmic Culture. University of Minnesota Press, Minneapolis (2006)

27. Garda, M.B.: Nostalgia in retro game design. In: Proceedings of the 2013 DiGRA International Conference: DeFragging Game Studies (2013)

28. Guckelsberger, C., Salge, C., Gow, J., Cairns, P.: Predicting player experience without the player: an exploratory study. In: Proceedings of the Annual Symposium on Computer-Human Interaction in Play, pp. 305-315 (2017). https://doi.org/10.1145/3116595. 3116631

29. Gutwin, C., Rooke, C., Cockburn, A., Mandryk, R.L., Lafreniere, B.: Peak-end effects on player experience in casual games. In: Proceedings of the 2016 CHI Conference on Human Factors in Computing Systems, pp. 5608-5619 (2016). https://doi.org/10. 1145/2858036.2858419

30. Harborth, D., Pape, S.: How nostalgic feelings impact Pokémon Go players-integrating childhood brand nostalgia into the technology acceptance theory. Behav. Inf. Technol. (2019). https://doi. org/10.1080/0144929X.2019.1662486

31. Iacovides, I., Cox, A., Kennedy, R., Cairns, P., Jennett, C.: Removing the HUD: the impact of non-diegetic game elements and expertise on player involvement. In: Proceedings of the Annual Symposium on Computer-Human Interaction in Play, pp. 13-22 (2015). https://doi.org/10.1145/2793107.2793120

32. IJsselsteijn, W.A., De Kort, Y.A. W., Poels, K.: The game experience questionnaire. Eindhoven: Technische Universiteit Eindhoven (2013)

33. Inal, Y., Cagiltay, K.: Flow experiences of children in an interactive social game environment. Br. J. Educ. Technol. 38(3), 455464 (2007). https://doi.org/10.1111/j.1467-8535.2007.00709.x

34. Johnson, D., Gardner, M.J., Perry, R.: Validation of two game experience scales: The Player Experience of Need Satisfaction (PENS) and Game Experience Questionnaire (GEQ). Int. J. Hum. Comput Stud. 118, 38-46 (2018). https://doi.org/10.1016/j.ijhcs. 2018.05.003

35. Johnson, D., Nacke, L.E., Wyeth, P.: All about that base: Differing player experiences in video game genres and the unique case of MOBA games. In: Proceedings of the 33rd Annual ACM Conference on Human Factors in Computing Systems, pp. 2265-2274 (2015) 
36. Johnson, D., Wyeth, P., Sweetser, P., Gardner, J.: Personality, genre and videogame play experience. In: Proceedings of the 4th International Conference on Fun and Games, pp. 117-120 (2012). https://doi.org/10.1145/2367616.2367633

37. Klarkowski, M., Johnson, D., Wyeth, P., McEwan, M., Phillips, C., Smith, S.: Operationalising and evaluating sub-optimal and optimal play experiences through challenge-skill manipulation. In: Proceedings of the 2016 CHI Conference on Human Factors in Computing Systems, pp. 5583-5594 (2016). https://doi.org/10. $1145 / 2858036.2858563$

38. Kokil, U., Sanchez, J.L.G.: Exploring facets of playability: the differences between PC and tablet gaming. In: Proceedings of the 8th International Conference on Advances in Computer-Human Interactions, pp. 108-111 (2015)

39. Law, E.L.C., Brühlmann, F., Mekler, E.D.: Systematic review and validation of the Game Experience Questionnaire (GEQ) - implications for citation and reporting practice. In: Proceedings of the Annual Symposium on Computer-Human Interaction in Play, pp. 257-270 (2018). https://doi.org/10.1145/3242671.3242683

40. Liszio, S., Emmerich, K., Masuch, M.: The influence of social entities in virtual reality games on player experience and immersion. In: Proceedings of the 12th International Conference on the Foundations of Digital Games, Article No: 35, pp. 1-10 (2017). https://doi.org/10.1145/3102071.3102086

41. Luo, Z., Guzdial, M., Liao, N., Riedl, M.: Player experience extraction from gameplay video. In: Proceedings of the Fourteenth Artificial Intelligence and Interactive Digital Entertainment Conference, pp. 52-58 (2018)

42. McLellan, S., Muddimer, A., Peres, S.C.: The effect of experience on system usability scale ratings. J. Usability Stud. 7(2), 56-67 (2012)

43. Mitgutsch, K.: Playful learning experiences: meaningful learning pattern in players' biographies. Int. J. Gaming Comput. Mediat. Simul. 3(3), 54-68 (2011). https://doi.org/10.4018/jgcms.20110 70104

44. Molde, H., Holmøy, B., Merkesdal, A.G., Torsheim, T., Mentzoni, R.A., Hanns, D., Pallesen, S.: Are video games a gateway to gambling? A longitudinal study based on a representative Norwegian sample. J. Gambl. Stud. 35(2), 545-557 (2019). https://doi.org/ 10.1007/s10899-018-9781-Z

45. Nacke, L., Mirza-Babaei, P., Drachen, A.: User experience (UX) research in games. In: Proceedings of the 2019 CHI Conference Extended Abstracts on Human Factors in Computing Systems, Paper No: C25, pp. 1-4 (2019). https://doi.org/10.1145/3290607. 3298826

46. Nacke, L., Schild, J., Niesenhaus, J.: Gameplay experience testing with playability and usability surveys - an experimental pilot study. In: Proceedings of the Fun and Games 2010 Workshop, NHTV Expertise Series, Vol. 10 (2010)

47. Nordin, A.I., Denisova, A., Cairns, P.: Too many questionnaires: measuring player experience whilst playing digital games. In: Proceedings of the Seventh York Doctoral Symposium on Computer Science and Electronics, pp. 69-74 (2014)

48. Norman, K.L.: GEQ (Game Engagement/Experience Questionnaire): a review of two papers. Interact. Comput. 25(4), 278-283 (2013). https://doi.org/10.1093/iwc/iwt009

49. Oksanen, K.: Subjective experience and sociability in a collaborative serious game. Simul. Gaming 44(6), 767-793 (2013). https:// doi.org/10.1177/1046878113513079

50. Philips, C.: Video game reward types \& the player experience. Unpublished doctoral dissertation, Queensland University of Technology (2018)

51. Pinelle, D., Wong, N., Stach, T.: Heuristic evaluation for games: usability principles for video game design. In: Proceedings of the
SIGCHI Conference on Human Factors in Computing Systems, pp. 1453-1462 (2008). https://doi.org/10.1145/1357054.1357282

52. Poels, K., De Kort, Y., Ijsselsteijn, W.: It is always a lot of fun! Exploring dimensions of digital game experience using focus group methodology. In: Proceedings of the 2007 Conference on Future Play, pp. 83-89 (2007). https://doi.org/10.1145/1328202. 1328218

53. Poels, K., De Kort, Y., IJsselsteijn, W.: Identification and categorization of digital game experiences: a qualitative study integrating theoretical insights and player perspectives. Westminst. Pap. Commun. Cult. 9(1), 107-129 (2012). https://doi.org/10.16997/ wpcc. 153

54. Rajanen, M., Nissinen, J.: A survey of game usability practices in Northern European game companies. In: Information Systems Research Seminar, Vol. 8 (2015)

55. Robinson, R., Murray, J., Isbister, K.: "You're Giving Me Mixed Signals!": a comparative analysis of methods that capture players' emotional response to games. In: Proceedings of the $2018 \mathrm{CHI}$ Conference Extended Abstracts on Human Factors in Computing Systems, Paper No: LBW567, pp. 1-6 (2018). https://doi.org/10. $1145 / 3170427.3188469$

56. Sabet, S.S., Griwodz, C., Möller, S.: Influence of primacy, recency and peak effects on the game experience questionnaire. In Proceedings of the 11th ACM Workshop on Immersive Mixed and Virtual Environment Systems, pp. 22-27 (2019). https://doi.org/ $10.1145 / 3304113.3326113$

57. Salen, K., Zimmerman,: Rules of Play. Game Design Fundamentals. The MIT Press, Cambridge (2004)

58. Sáenz-de-Urturi, Z.G., Zapirain, B., Méndez Zorrilla, A.: Elderly user experience to improve a kinect-based game playability. Behav. Inf. Technol. 34(11), 1040-1051 (2015). https://doi.org/ 10.1080/0144929X.2015.1077889

59. Sánchez, J.L.G., Padilla, Z.N., Gutiérrez, F.L.: From usability to playability: Introduction to player-centred video game development process. In: Kurosu, M. (ed.) Human Centered Design. HCD 2009 Lecture Notes in Computer Science, vol. 5619. Springer, Berlin (2009). https://doi.org/10.1007/978-3-642-02806-9_9

60. Seif El-Nasr, M., Desurvire, H., Nacke, L., Drachen, A., Calvi, L., Isbister, K., Bernhaupt, R.: Game user research. In: Proceedings of the 2012 CHI Conference Extended Abstracts on Human Factors in Computing Systems, pp. 2679-2682 (2012). https://doi. org/10.1145/2212776.2212694

61. Shaker, N., Asteriadis, S., Yannakakis, G.N., Karpouzis, K.: A game-based corpus for analysing the interplay between game context and player experience. In: D'Mello, S., Graesser, A., Schuller, B., Martin, J.C. (eds.) Affective Computing and Intelligent Interaction. ACII 2011. Lecture Notes in Computer Science, vol. 6975. Springer, Berlin (2011). https://doi.org/10.1007/978-3642-24571-8_68

62. Shields, A.B., Johnson, J.W.: Childhood brand nostalgia: a new conceptualization and scale development. J. Consum. Behav. 15(4), 359-369 (2016). https://doi.org/10.1002/cb.1578

63. Sim, G., MacFarlane, S., Read, J.: All work and no play: measuring fun, usability, and learning in software for children. Comput. Educ. 46(3), 235-248 (2006). https://doi.org/10.1016/j.compedu. 2005.11.021

64. Siqueira, E.S., Santos, T.A.A., Castanho, C.D., Jacobi, R.P.: Estimating player experience from arousal and valence using psychophysiological signals. In: Proceedings of the 17th Brazilian Symposium on Computer Games and Digital Entertainment, pp. 107-116 (2018). https://doi.org/10.1109/SBGAMES.2018.00022

65. Smeddinck, J.D., Mandryk, R.L., Birk, M.V., Gerling, K.M., Barsilowski, D., \& Malaka, R.: How to present game difficulty choice?: Exploring the impact on player experience. In: Proceedings of the 2016 CHI Conference on Human Factors in Computing 
Systems, pp. 5595-5607 (2016). https://doi.org/10.1145/2858036. 2858574

66. Time Magazine. The ultimate game freak. http://content.time.com/ time/magazine/article/0,9171,2040095,00.html (1999). Accessed Jan 2021

67. Tractinsky, N., Katz, A.S., Ikar, D.: What is beautiful is usable. Interact. Comput. 13(2), 127-145 (2000). https://doi.org/10.1016/ S0953-5438(00)00031-X

68. Tullis, T., Albert, W.: Measuring the User Experience: Collecting, Analyzing, and Presenting Usability Metrics. Morgan Kaufmann, Burlington (2008)

69. Venkatesh, V., Thong, J., Xu, X.: Consumer acceptance and user of information technology: extending the unified theory of acceptance and use of technology. MIS Q. 36(1), 157-178 (2012). https://doi.org/10.2307/41410412

70. Wake, J.D., Guribye, F., Wasson, B.: Learning through collaborative design of location-based games. Int. J. Comput. Support.
Collab. Learn. 13(2), 167-187 (2018). https://doi.org/10.1007/ s11412-018-9278-x

71. Whalen, Z., Taylor, L.N.: Playing the past. An introduction. Playing the past. In: Whalen, Z., Taylor, L.N. (eds.) History and Nostalgia in Video Games. Vanderbilt University Press, Nashville (2008)

72. Wulf, T., Bowman, N.D., Velez, J.A., Breuer, J.: Once upon a game: exploring video game nostalgia and its impact on wellbeing. Psychol. Pop. Media Cult. 9(1), 83-95 (2018). https://doi. org/10.1037/ppm0000208

Publisher's Note Springer Nature remains neutral with regard to jurisdictional claims in published maps and institutional affiliations. 